\title{
Caracterização biogeoquímica da matéria orgânica em um estuário de macromaré localizado na interface Amazônia-semiárido no nordeste do Brasil
}

\author{
Mariana Costa Corrêa ${ }^{1,3}$ \\ Jefferson Horley Feitosa Serejo ${ }^{1}$ \\ Thiago Pessanha Rangel ${ }^{2}$ \\ Braulio Cherene Vaz de Oliveira ${ }^{2}$ \\ Marcelo Gomes de Almeida ${ }^{2}$ \\ Francisco José da Silva Dias ${ }^{1}$ \\ Carlos Eduardo de Rezende ${ }^{2,}$ \\ ${ }^{1}$ Laboratório de Hidrodinâmica Costeira, \\ Estuarina e de Águas Interiores - LHiCEAI \\ Universidade Federal do Maranhão \\ Avenida dos Portugueses, s/n - Bacanga \\ São Luís, MA, Brasil \\ CEP $65085-580$ \\ ${ }^{2}$ Laboratório de Ciências Ambientais - LCA \\ Universidade Estadual do Norte \\ Fluminense Darcy Ribeiro \\ Avenida Alberto Lamego, 2000, Parque \\ Califórnia \\ Campos dos Goytacazes, RJ, Brasil \\ CEP 28013602 \\ ${ }^{3}$ Endereço Atual \\ Laboratório de Geoquímica Orgânica \\ Universidade Federal Fluminense \\ Instituto de Química - $4^{\circ}$ andar - Outeiro São \\ João Baptista s/n \\ Centro, Niterói, RJ, Brasil \\ CEP: 24.020-141 \\ *Autores de correspondência \\ marianacorrea54@gmail.com \\ crezende@uenf.br
}

\section{RESUMO}

Os estuários são ambientes costeiros de transição, que atuam como um filtro natural e sítio de troca de água e matéria, principalmente matéria orgânica, onde grande parte do carbono pode ser importado e/ou exportado. O objetivo deste estudo é compreender a composição da matéria orgânica na coluna de água do Complexo Estuarino da Baía de São Marcos, um ambiente sob a influência de macromaré semi-diurna. Para traçar a distribuição da matéria orgânica com mais precisão, a composição elementar é usada juntamente com a composição isotópica do carbono e do nitrogênio. A amostragem foi realizada em agosto de 2016, em três transectos, totalizando 14 estações. A água foi amostrada em duas profundidades diferentes, subsuperficie e fundo. Os seguintes parâmetros foram medidos: material particulado em suspensão, carbono orgânico dissolvido, carbono orgânico particulado, isótopos estáveis de carbono e nitrogênio, razão atômica $(\mathrm{C} / \mathrm{N})$ a. Os parâmetros condutividade elétrica, oxigênio dissolvido e temperatura foram medidos in situ. O material particulado em suspensão teve média de $360,6 \mathrm{mg} \mathrm{L}^{-1}$ ) no meio com uma pequena diminuição (média de $344,6 \mathrm{mg} \mathrm{L}^{-1}$ ) para a parte mais interna da baía. O carbono orgânico apresentou maiores concentrações sob a forma dissolvida (média 10,97 $\mathrm{mg} \mathrm{L}^{-1}$ ), variando entre as profundidades e os pontos amostrais. As concentrações de nitrogênio total foram baixas (média $0,38 \mathrm{mg} \mathrm{L}^{-1}$ ), estando relacionadas à pouca influência de compostos nitrogenados. A razão isotópica e $\mathrm{C} / \mathrm{N}$ indicam que o manguezal representa uma importante fonte de carbono orgânico particulado e dissolvido nesse estuário.

Palavras-chave: Complexo estuarino, marcadores geoquímicos, carbono orgânico, isótopos estáveis, Complexo Estuarino da Baía de São Marcos

\section{ABSTRACT}

Estuaries are transitional, coastal environments that act as natural filters and areas for the exchange of water and materials, mainly organic matter, which is composed of a significant portion of the transported carbon. Estuaries may import, export or retain dissolved and particulate organic matter. The aim of this study is to understand the composition of the organic matter in the water column of São Marcos Bay Estuarine Complex, a macrotidal environment under influence of semidiurnal tides. To investigate the distribution of organic matter more precisely, the elemental composition was used along with the carbon and nitrogen isotopic composition. The sampling was carried out in August 2016, in 3 transects, with a total of 14 stations. The water was sampled in two different depths, subsurface and bottom. The following parameters were measured: suspended particulate matter, dissolved organic carbon, particulate organic carbon, carbon and nitrogen stable isotopes andthe atomic ratio $(\mathrm{C} / \mathrm{N}) \mathrm{a}$. The parameters electrical conductivity, dissolved oxygen, and temperature were measured in situ. SPM had a mean of 360.6 $\left(\mathrm{mg} \mathrm{L}^{-1}\right)$ in the medium with a small decrease (mean of $344.6 \mathrm{mg} \mathrm{L}^{-1}$ ) for the innermost part of the bay. The organic carbon presented higher concentrations in the dissolved mean $10.97 \mathrm{mg} \mathrm{L}^{-1}$ form than the particulate form mean $0.38 \mathrm{mg} \mathrm{L}^{-1}$, varying between the depths and the sampling 
stations. The total nitrogen concentrations were low and may be related to the low influence of nitrogen compounds. The isotopic signatures and the $\mathrm{C} / \mathrm{N}$ ratios indicated that mangroves are the most abundant source of particulate and dissolved organic carbon in the study region.

Keywords: estuarine complex, geochemical tracers, stable isotope, carbon, nitrogen, São Marcos Bay Estuarine Complex.

\section{INTRODUÇÃO}

A zona costeira representa uma região de transição e exerce uma importante função de interação entre os ecossistemas marinhos e terrestres. Dentre os ambientes costeiros, estão os estuários. Há considerável embate sobre a definição de um estuário por causa das propriedades divergentes encontradas dentro do ecossistema em diferentes regiões do mundo. São comumente descritos como corpos de água semifechados, situados na interface entre o continente e o oceano, onde a água do mar é mensuravelmente diluída pela entrada de água doce (Hobbie 2000).

Em condições naturais, os estuários são regiões biologicamente mais produtivas que os rios e o oceano, recebem grandes quantidades de matéria orgânica, nutrientes, sedimentos e alguns recebem contaminantes (Canuel 2001). Esse ecossistema dinâmico possui a maior diversidade e produção biótica do mundo, além de desempenhar um papel importante na biogeoquímica do carbono nos sistemas aquáticos (Miranda et al. 2002). Os ciclos biogeoquímicos que envolvem a interação entre os processos biológicos, químicos $\mathrm{e}$ geológicos determinam fontes, sumidouros e fluxos de elementos através de diferentes reservatórios dentro dos ecossistemas (Bianchi 2006).

A matéria orgânica nos ambientes estuarinos, em geral, é derivada de uma multiplicidade de fontes alóctones (aquela produzida fora do ecossistema), trazidas pelo escoamento superficial, rios, floresta de mangue e oceano aberto. E fontes autóctones, matéria orgânica produzida dentro do próprio sistema, por meio da produção primária (Rezende et al. 1990, Goñi et al. 2003, Canuel\& Hardison 2016). O caráter geoquímico desses materiais quando alcançam o oceano adjacente é significativamente diferente do original, pois os estuários são, ao mesmo tempo, um "filtro e um reator" biogeoquímico, transformando a matéria orgânica e inorgânica derivada da captação, e regulando a liberação de carbono para plataforma continental (Baueret al. 2013; Liuet al. 2015).

De uma maneira histórica e global, os estuários vêm sofrendo grandes pressões através do desenvolvimento urbano, agrícola, industrial, turístico e recreacional. O reflexo disso é a dimuição dos manguezais. De acordo com Giri et al. (2011) nas últimas três décadas, as perdas florestais por fatores antropogênicos aumentaram significativamente, e a área restante da floresta de manguezais no mundo é inferior ao que se pensava anteriormente, estima-se que é $12,3 \%$ menor do que a estimativa mais recente feita pela Organização das Nações Unidas para Agricultura e Alimentação (FAO 2007). O seu rápido desaparecimento e degradação causarão consequências negativas no balanço de carbono podendo influenciar a composição atmosférica e o clima (Giri et al. 2011). A compreensão aprimorada do ciclo do carbono nessas áreas é importante para compreender e prever o papel da região costeira na absorção de $\mathrm{CO}_{2}$ antropogênico da atmosfera (Hedges et al. 1997).

A matéria orgânica, onde está grande parte do carbono transportado, pode ter diferentes formas e tamanhos, ser constituída por inúmeros compostos químicos, que consiste basicamente de detritos vegetais e animais, formados por proteínas, carboidratos, lipídios, lignina, por material refratário constituído por ácidos húmicos e fúlvicos (Abrantes et al. 2014, Hermes\& Sikes 2016). A matéria orgânica dissolvida (MOD) é a principal forma de matéria orgânica em todos os ecossistemas aquáticos (Mctiernan et al. 2001, Hertkon et al. 2002).

De modo a traçar de forma mais precisa a origem desse material, normalmente são conciliados o uso da composição isotópica, elementar e biomarcadores, uma vez que em ecossistemas complexos a multiplicidade de fontes não permite que um só traçador determine com segurança a origem do material presente em uma determinada fração. Além de 
possuírem grande importância no processo de identificação de fontes, estas ferramentas biogeoquímicas possibilitam também a geração de informações sobre o estado de alteração da matéria orgânica (Fry 1981, Bouillon et al. 2008, Souza et al. 2017).

A utilização de isótopos estáveis é um método adequado, principalmente, pelo fato de estas assinaturas serem preservadas relativamente bem (ou modificadas dentro de limites aceitáveis) durante a mineralização, transporte e deposição (Kristensen et al. 2008, Bouillon et al. 2011). Assim, os isótopos estáveis oferecem inúmeras possibilidades de monitorar a origem e transformações no ambiente aquático, tais como uso da terra, processos geoquímicos, acumulação e absorção na cadeia trófica. Junto à composição isotópica usa-se a elementar, pois são ferramentas necessárias para compreender $\mathrm{o}$ ciclo da matéria orgânica e detectar as possíveis causas das mudanças ambientais (Petersonet al. 1985, Fry 1981, Rezendeet. al. 1990, Liuet al. 2015). A utilização dos isótopos estáveis de carbono e nitrogênio, apartir das razões isotópicas entre $13 \mathrm{C} / 12 \mathrm{C}$ e $15 \mathrm{~N} / 14 \mathrm{~N}$,na diferenciação entre fontes de matéria orgânica é baseada na ampla variabilidade das razões isotópicas (Brintow 2013). A composição elementar é adequada para análises de fontes, pois possibilita distinguir padrões de abundância de elementos biogênicos em diferentes tipos de organismos (Hedges 1990). As relações existentes entre os diferentes constituintes envolvidos na composição elementar da matéria orgânica podem indicar qualitativamente as fontes envolvidas (Moyer et at. 2013).

A matéria orgânica de origem terrestre, proveniente de plantas de metabolismo C3, possui razão isotópica entre -24 a $-38 \%$, enquanto plantas de metabolismo via $\mathrm{C} 4$,

\section{2. ÁREA DE ESTUDO}

O Complexo Estuarino de São Marcos (CESM), popularmente conhecido como Baía de São Marcos (BSM) é uma vasta zona estuarina, que recebe águas de uma importante bacia de drenagem (Rio Mearim) localizado no nordeste do Brasil (Figura 1), com orientação principal NE-SW, amplamente aberta sobre a plataforma continental. A bacia do rio Mearim que deságua no CESM é a maior bacia de drenagem do estado do Maranhão com área de $99.059 \mathrm{~km}^{2}$, correspondendo aproximadamente $30 \%$ da área total do estado. Esta bacia possui possuem uma faixa entre -11 a $-15 \%$ (Martinelli et al, 2009). Entretanto, os valores típicos de $\delta^{13} \mathrm{C}$ da matéria orgânica marinha, produzida principalmente por fitoplâncton variam de -22 a $-18 \%$ (Meyers 1997) e a mistura entre matéria orgânica marinha e terrestre possui um $\delta^{13} \mathrm{C}$ de $-23 \%$ (Pancost \& Boot 2004), enquanto a vegetação e sedimentos de manguezal variam de -25 a 28\% (Lacerda et al. 1986; Bouillon et al. 2008). A composição isotópica do nitrogênio estável nas plantas não segue um padrão tão bem definido quanto à do carbono. $\mathrm{O} \delta^{15} \mathrm{~N}$ das plantas depende em grande parte da fonte de nitrogênio $\left(\mathrm{N}_{2}\right.$ atmosférico ou inorgânico) (Martinelli et al. 2009, Maya et al. 2011).

Devido às sobreposições encontradas nos ecossistemas costeiros a razão atômica $(\mathrm{C} / \mathrm{N}) \mathrm{a}$ pode ser utilizada como indicador potencial para elucidar a matéria orgânica marinha e terrestre. A razão entre 6 e 10 caracteriza a matéria orgânica de origem marinha, razão $\mathrm{C} / \mathrm{N}$ maior que 20 pode indicar que compostos terrestres são principais fontes de MO para o sistema (Meyers 1994, Meyers 1997, Bianchi 2006). A relação entre as assinaturas de isótopos estáveis de manguezais e fatores ambientais é bastante complexa (Bouillon 2008). Mesmo assim, a composição elementar e isotópica torna-se uma importante ferramenta para indicar possíveis fontes da matéria orgânica nos sistemas costeiros.

Dessa maneira, o objetivo desse estudo é saber, por meio da composição elementar e isotópica como a matéria orgânica nas frações particulada e dissolvida transportadas se comporta nesse complexo estuarino, localizado na zona costeira do Maranhão. Assim, pretende-se determinar as principais fontes de matéria orgânica para o ambiente e se de fato ela é exportada ou retida no sistema.

cerca de 1,6 milhões de habitantes sendo que menos de $20 \%$ dos esgotos domésticos são tratados. Sob o ponto de vista hidrodinâmico a costa do estado do Maranhão é classificada como sendo do tipo macromaré, sob a ação constante dos ventos alísios. Com marés do tipo semidiurnas, com preamares que chegam até $7,2 \mathrm{~m}$ de altura nas grandes sizígias, com média de $6,6 \mathrm{~m}$, correntes fortes de maré, chegando a $2,5 \mathrm{~m} \mathrm{~s}^{-1}$ (El-Robrini et al. 2006). 
No estado do Maranhão o clima é influenciado por dois principais sistemas de circulação, geradores de tempos instáveis: a Zona de Convergência Intertropical (ZCIT) e as "linhas" de Instabilidade Tropical (IT) isso acaba influenciando na sazonalidade de chuvas (Luz et al. 2005). No Maranhão os índices de precipitação pluviométrica são elevados, ainda mais quando comparado ao contexto nordestino. Os valores variam de $1000 \mathrm{~mm}$ a
$2000 \mathrm{~mm}$ distribuídos principalmente nos meses de janeiro a junho, meses da estação chuvosa, no qual março, abril e maio encontram-se as frequências mais altas. Já nos meses de julho a dezembro os níveis de precipitação diminuem com as menores médias pluviométricas mensais que ocorrem em setembro, outubro e novembro, caracterizando como período de estiagem (Araújo 2017, Teixeira \& Souza Filho 2009).
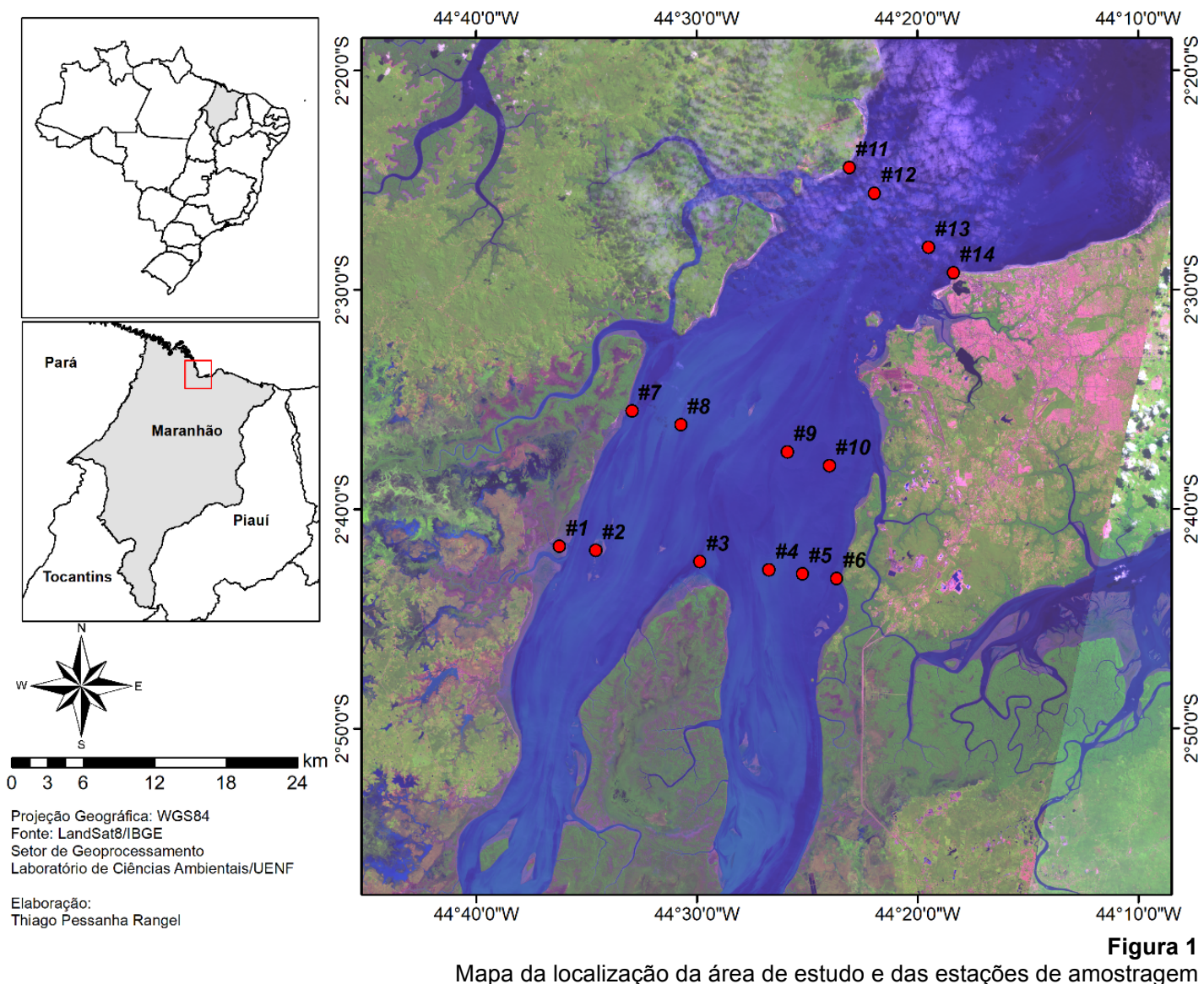

\section{MATERIAIS E MÉTODOS}

As amostragens no CESM ocorreram durante a estação de seca, agosto de 2016, em evento de maré de quadratura e realizadas com o auxílio da garrafa de Niskin, sendo divididas em água superficial $(0,5 \mathrm{~m}$ abaixo da linha d'água; $\mathrm{n}=14)$ e fundo $(0,5 \mathrm{~m}$ acima do substrato de fundo; $n=14$ ), para que não tocasse o fundo foi tomado o cuidado fazendo uma medição prévia da profundidade local com a ajuda dos equipamentos da embarcação e como a corda da garrafa era marcada a cada um metro, o cabo utilizado sempre ia de um metro a meio metro acima do limite total, distribuídos em 3 transectos longitudinais (Figura 1). As amostras foram armazenadas em garrafas previamente descontaminadas, permanecendo resfriadas durante todo $\mathrm{o}$ período de coleta, para conservação de suas propriedades. Em uma de correntes menos intensas foram realizadas coletas de material particulado em suspensão, folha de mangue e sedimento de fundo $(<2,0 \mathrm{~mm})$ (Serejo 2018).

Para a concentração do carbono orgânico dissolvido (COD) as amostras foram filtradas (filtros $\mathrm{GF} / \mathrm{F}$ com porosidade nominal de $0,7 \mu \mathrm{m})$, em bomba a vácuo, em três alíquotas de $40 \mathrm{ml}$, armazenadas em frascos de vidro âmbar contendo $1 \%(\mathrm{v} / \mathrm{v})$ de $\mathrm{H}_{3} \mathrm{PO}_{4} 10 \%$, sendo armazenadas a $4^{\circ} \mathrm{C}$ em geladeira até análise no Laboratório de Ciências Ambientais da Universidade Estadual do Norte Fluminense. Os parâmetros temperatura, salinidade, condutividade e $\mathrm{pH}$ foram obtidos in situ com o auxílio de uma sonda 
multiparamétrica HANNA-HI98194/10. O MPS presente nas águas da Baía de São Marcos foi determinado por medida gravimétrica, segundo as metodologias descritas em Strickland \& Parsos (1972). A matéria orgânica dissolvida foi isolada das amostras por meio da técnica de extração de fase sólida (Dittmar 2008). As amostras filtradas foram passadas por gravidade nos cartuchos de extração de fase sólida (5g PPL, Agilent) ativados com $40 \mathrm{~mL}$ de metanol (grau HPLC), dessalinizados usando $80 \mathrm{~mL}$ de solução, água ultrapura a (pH2), 0,01 $\mathrm{molL}^{-1}$, secos em fluxo de $\mathrm{N}_{2}$ e eluídos com $40 \mathrm{~mL}$ de metanol (grau de pureza HPLC). A eficiência da extração do COD foi determinada para cada amostra após evaporação de uma alíquota de $200 \mu \mathrm{L}$ do extrato obtidos das colunas utilizadas e, em média, a recuperação foi de aproximadamente $100 \%$ do COD $(n=28)$. A determinação da composição elementar e isotópica da MOD foi realizada com a retirada de 1000 a $2500 \mu \mathrm{L}$ dos extratos de metanol

\section{RESULTADOS E DISCUSSÃO}

\subsection{CLIMA E PARÂMETROS FÍSICO-QUÍMICOS}

A influência dos afluentes sob o CESM é percebida através do gradiente de condutividade no contínuo continente-oceano. O volume total da precipitação pluviométrica (dados INMET), para o período em questão, foi de $13,4 \mathrm{~mm}$ e a velocidade do vento foi de $2,5 \mathrm{~m} \mathrm{~s}^{-1}$.

A tabela 1 (anexo) mostra a variação dos parâmetros físico-químicos mensurados na coluna de água em subsuperfície no fundo. Apesar da homogeneidade vertical da coluna de água houve aumento da condutividade, com estratificação apenas nas estações 7,8 e 9 do transecto 2 podendo ser atribuído aos pequenos rios que deságuam na área adjacente. A temperatura da água esteve acima de $28^{\circ} \mathrm{C}$, resultante da mistura das massas de água
(40mL) com a matéria orgânica dissolvida das amostras Para as análises MOP os filtros foram postos em atmosfera ácida, utilizando $\mathrm{HCl}$ concentrado, durante $48 \mathrm{~h}$ e em seguida transferidos para forno mufla por $4 \mathrm{~h}$ a $110^{\circ} \mathrm{C}$, para total secura. A composição elementar $(\mathrm{C}$ e $\mathrm{N})$ e a isotópica $\left(\delta^{13} \mathrm{C}\right.$ e $\left.\delta^{15} \mathrm{~N}\right)$ foi determinada através do equipamento Flash 2000 (Organic Elemental Analyzer - ThermoScientific) acoplado à interface Conflo IV com um espectrômetro de massas Delta V Advantage (IsotopeRatio Mass Spectrometer, IRMS ThermoScientific). A razão isotópica foi obtida como descrito abaixo:

$$
\delta^{N} E . \% 0=\left[\frac{R_{\text {amostras }}}{R_{\text {padrão }}}-1\right] X 100
$$

onde $\mathrm{N}$ é o isótopo mais pesado do elemento $\mathrm{E}$ (carbono ou nitrogênio) e $\mathrm{R}$ é a razão do isótopo mais pesado sobre o mais leve $\left({ }^{13} \mathrm{C} /{ }^{12} \mathrm{C},{ }^{15} \mathrm{~N} /{ }^{14} \mathrm{~N}\right)$ desses elementos.

\subsection{MATERIAL PARTICULADO EM SUSPENSÃO, CARBONO PARTICULADO E NITROGÊNIO PARTICULADO}

$\mathrm{Na}$ água superficial o material particulado em suspensão (MPS) variou de 59,95 a 541,47 $\mathrm{mg} \mathrm{L}^{-1}$ e no fundo foi de 77,17 a $750,18 \mathrm{mg} \mathrm{L}^{-}$ ${ }^{1}$.Há um aumento de MPS de $27 \%$ no fundo, sugerindo que a entrada de massa de água costeira (AC) ressuspende o material recém depositado e/ou barra hidraulicamente os costeira e continental (Dias et al, 2013). No período de seca, há uma clara diminuição dos fluxos fluviais e um potencial aumento do domínio de águas costeiras sobre o CESM, que fica evidenciado em eventos de maré de quadratura, como apontam os dados deste estudo, e corrobora os resultados obtidos por Lefvre et al. (2017).

As características ambientais implicam diretamente nos processos de entrada, modificações, deposição e exportação da matéria orgânica particulada e dissolvida (MOP e MOD, respectivamente) para o ambiente adjacente e abaixo estaremos apresentando os resultados obtidos neste estudo 
Tabela 1 - Valores de profundidade de coleta, temperatura, condutividade, oxigênio dissolvido e localização dos pontos em cada transecto.

\begin{tabular}{|c|c|c|c|c|c|}
\hline Regiões & $\begin{array}{c}\text { Pontos } \\
\text { Amostrais }\end{array}$ & $\begin{array}{c}\text { Profundidade } \\
\text { (m) }\end{array}$ & $\begin{array}{c}\text { Temperatura } \\
\left({ }^{\circ} \mathrm{C}\right)\end{array}$ & $\begin{array}{c}\text { Condutividade } \\
\left(\mathrm{mS}^{2} \mathrm{~cm}^{1}\right)\end{array}$ & $\begin{array}{c}\text { Oxigênio Dissolvido } \\
\left(\mathrm{mg}^{\left.-\mathrm{L}^{-1}\right)}\right.\end{array}$ \\
\hline \multirow{6}{*}{ Transecto 1} & 1 & 0,5 & 29,0 & 42940 & 3,3 \\
\hline & 2 & 0,5 & 29,3 & 43980 & 4,2 \\
\hline & 3 & 0,5 & 29,1 & 43940 & 3,7 \\
\hline & 4 & 0,5 & 28,9 & 44930 & 4,5 \\
\hline & 5 & 0,5 & 28,9 & 47360 & 4,4 \\
\hline & 6 & 0,5 & 28,6 & 47100 & 4,3 \\
\hline \multirow{4}{*}{ Transecto 2} & 7 & 0,5 & 28,1 & 21640 & 4,3 \\
\hline & 8 & 0,5 & 28,6 & 33830 & 6,1 \\
\hline & 9 & 0,5 & 28,6 & 20270 & 6,3 \\
\hline & 10 & 0,5 & 28,9 & 47540 & 5,6 \\
\hline \multirow{4}{*}{ Transecto 3} & 11 & 0,5 & 28,9 & 51500 & 4,9 \\
\hline & 12 & 0,5 & 28,3 & 49700 & 4,9 \\
\hline & 13 & 0,5 & 28,7 & 52200 & 4,8 \\
\hline & 14 & 0,5 & 28,1 & 52650 & 4,6 \\
\hline \multirow{6}{*}{ Transecto 1} & 1 & 3 & 29,0 & 43200 & 3,3 \\
\hline & 2 & 2 & 330,6 & 43890 & 3,9 \\
\hline & 3 & 2 & 28,9 & 43730 & 4,2 \\
\hline & 4 & 6 & 29,1 & 45210 & 4,0 \\
\hline & 5 & 11 & 28,9 & 46070 & 3,8 \\
\hline & 6 & 10 & 28,7 & 45900 & 3,2 \\
\hline \multirow{4}{*}{ Transecto 2} & 7 & 8 & 28,7 & 43330 & 3,0 \\
\hline & 8 & 14 & 28,9 & 44440 & 4,8 \\
\hline & 9 & 13 & 28,4 & 46970 & 5,5 \\
\hline & 10 & 5 & 28,6 & 47310 & 5,2 \\
\hline \multirow{4}{*}{ Transecto 3} & 11 & 8 & 28,5 & 52300 & 5,2 \\
\hline & 12 & 23 & 28,3 & 51400 & 4,8 \\
\hline & 13 & 15 & 28,3 & 52400 & 4,9 \\
\hline & 14 & 6 & 28,1 & 52800 & 5,3 \\
\hline
\end{tabular}

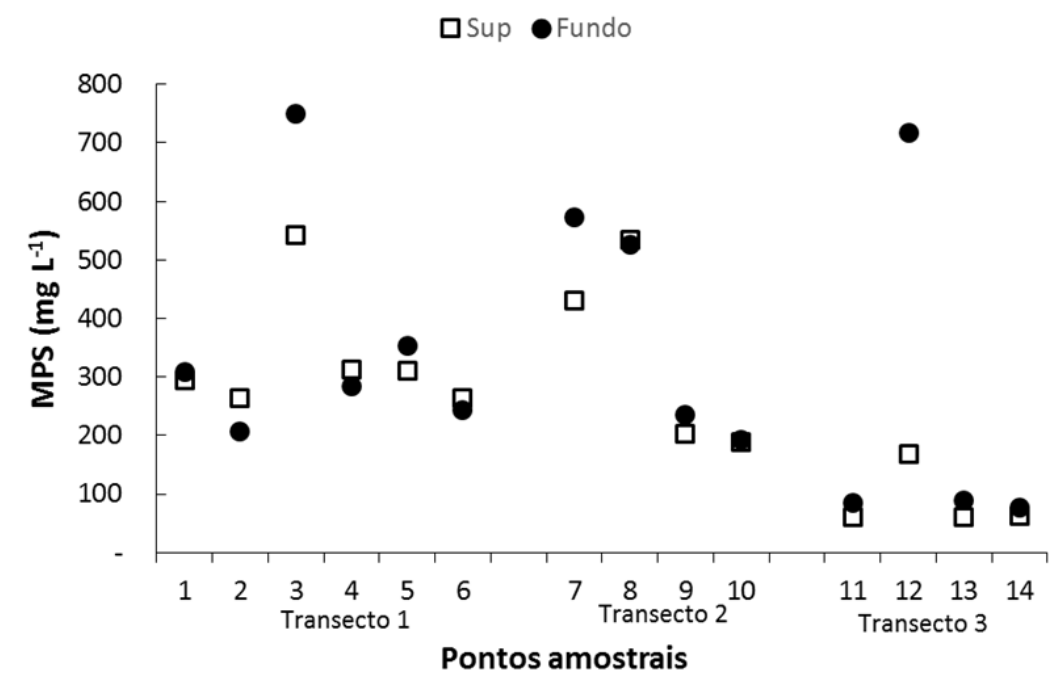

Figura 2

Distribuição do material particulado em suspensão ao longo dos três transectos, profundidades e pontos de coleta. Com maiores concentrações a montante do estuário (transecto1) diminuído a concentração com aumento dos sais mais a jusante

(transecto3).

As maiores concentrações médias de carbono orgânico $\left(8,49 \mathrm{mg} \mathrm{L}^{-1}\right)$ e nitrogênio total particulado $\left(0,90 \mathrm{mg} \mathrm{L}^{-1}\right)$, (COP e NTP, respectivamente) ocorreram sempre no fundo e assim como na superfície diminuíram à medida que se afastava da costa $\left(8,79 \mathrm{mg} \mathrm{L}^{-1}, 9,78 \mathrm{mg}\right.$ $\mathrm{L}^{-1}$ e $6,77 \mathrm{mg} \mathrm{L}^{-1}$ para o COP; e $0,92 \mathrm{mg} \mathrm{L}^{-1}$, $1,04 \mathrm{mg} \mathrm{L}^{-1}, 0,73 \mathrm{mg} \mathrm{L}^{-1}$ para COP e NTP respectivamente) (Figura 3). O COP e NTP ao longo dos transectos variou de 1,79 a $13,17 \mathrm{mg}$ $\mathrm{L}^{-1}$ e 0,20 a $1,00 \mathrm{mg}^{-1}$ na superfície, respectivamente, enquanto no fundo foram observadas as maiores variações das concentrações (2,16 a 23,32 $\mathrm{mg} \mathrm{L}^{-1}$ e o NTP de 0,20 a $2,46 \mathrm{mg} \mathrm{L}^{-1}$ ), retratando a ressuspensão 
de material fino depositado nos sedimentos de fundo.

A entrada de massa de água costeira (AC) e as correntes impulsionadas pelo vento são as principais variáveis que influenciam o transporte e a mistura do MPS, COP e NTP na coluna de água. Estes parâmetros exibiram um comportamento não conservativo com uma perda expressiva em relação ao aumento da condutividade elétrica, sugerindo que o estuário inferior adentra continente acima na estação seca tornando-se um retentor de sedimentos. A grande diferença de densidade entre massas de água costeira (AC) e continental (água do rio) provoca um barramento hidráulico dos sedimentos carreados pela drenagem continental, acarretando a formação de novas ilhas de sedimentação e perda de profundidade local. Os estudos de Corrêa (2016) na mesma região corroboram os dados mensurados, onde pode ser observada uma baixa variabilidade nas concentrações do MPS nos perfis verticais e diminuição com o aumento da salinidade, esse fato foi atribuído à grande exportação de partículas em suspensão pelo rio Mearim, forte interação da maré em toda coluna d'água, sedimentação e floculação induzida pela salinidade e retenção de materiais dentro do ambiente. $\mathrm{O}$ barramento hidráulico provocado pelo avanço de massa de água costeira aumenta as taxas de deposição do MPS, devido aos processos de floculação que alteram o tamanho de partícula, densidade e velocidade de sedimentação (Eckert \& Sholkovitz 1976, Mikkelsen et al. 2007).

A influência sazonal da precipitação e das marés são fatores determinantes no transporte de material na interface continente-oceano, onde nas estações do ano de menor fluxo fluvial a influência da massa de água marinha se desloca estuário interno e acima. Entretanto, em períodos de maiores vazões fluviais, como consequência, temos um aumento de aporte de materiais para a plataforma continental adjacente, contribuindo com o balanço global de carbono para o Atlântico Equatorial. Este comportamento tem sido descrito por vários autores em diferentes regiões e destacamos os estudos de Hermes \& Sikes (2016) no Rio Delaware e Souza et al. (2010) no Rio Paraíba do Sul, dois sistemas com diferentes tipologias fluviais e climáticas.

\subsection{CARBONO ORGÂNICO DISSOLVIDO E NITROGÊNIO TOTAL DISSOLVIDO}

A distribuição do carbono orgânico dissolvido (COD) em relação ao gradiente salino é apresentada na figura3. Nos transectos 2 e 3 as concentrações de COD variaram de 3,13 a $4,62 \mathrm{mg} \mathrm{L}^{-1}$, e no transecto 1 as concentrações estiveram acima de $6,92 \mathrm{mg} \mathrm{L}^{-1}$ sendo observadas variações nas profundidades de coleta, principalmente, nos pontos 1,2 e 3 $\left(13,25\right.$ a $20,29 \mathrm{mg} \mathrm{L}^{-1} ; 6,92$ a $44,63 \mathrm{mg} \mathrm{L}^{-1} \mathrm{e}$ 10,83 a $62,74 \mathrm{mg} \mathrm{L}^{-1}$, respectivamente). $\mathrm{O}$ nitrogênio total dissolvido (NTD) variou de 0,22 a $0,52 \mathrm{mg} \mathrm{L}^{-1}$ na superfície a montante e os pontos mais próximos à conexão com o mar aberto tiveram concentrações menores $(0,24$ a $\left.0,35 \mathrm{mg} \mathrm{L}^{-1}\right)$ e nos demais pontos de coleta as concentrações ficaram entre 0,34 a $0,50 \mathrm{mg} \mathrm{L}^{-}$ 1, tipificando um ambiente pobre em compostos nitrogenados (Figura 4).A fração dissolvida do carbono orgânico foi superior ao COP na superfície e fundo, enquanto as concentrações de nitrogênio total dissolvido foram menores do que na fração particulada. As concentrações de nitrogênio total.
As altas concentrações de COD (44,7 mg L ${ }^{1}$ e $62,7 \mathrm{mg} \mathrm{L}^{-1}$ ) foram medidas em locais de baixa profundidade e formação de ilha de deposição à frente dessa região que ocasionou o aprisionamento do carbono. Com o avanço da intrusão de água marinha houve uma liberação desse carbono dissolvido armazenado gerando estes pulsos de material orgânico dissolvido. As maiores concentrações de material orgânico dissolvido no CESM são atribuídas à circulação interna deste sistema assim como pela proximidade das extensas áreas de manguezais. Esse ecossistema costeiro, úmido é característico da área estudada, principalmente por conta da densa cobertura das florestas de manguezal, o que acaba potencializando aporte e dinâmica do carbono para as áreas costeiras adjacentes. Vários estudos têm descrito a importância dos manguezais no aporte de carbono para as áreas costeiras adjacentes (Dittmar et al. 2006, Rezende et al. 2007, Tremblay et al. 2007). 


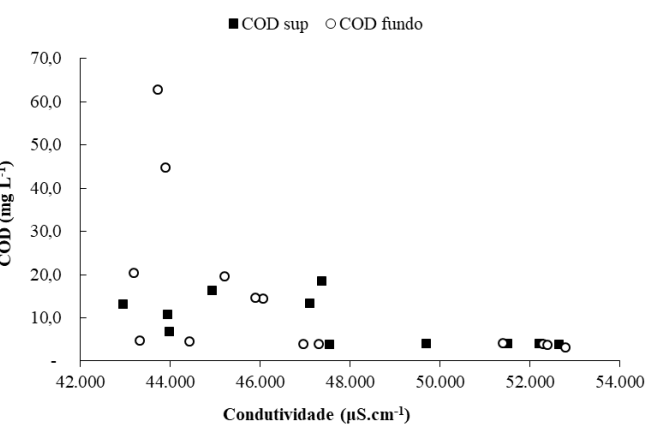

(a)

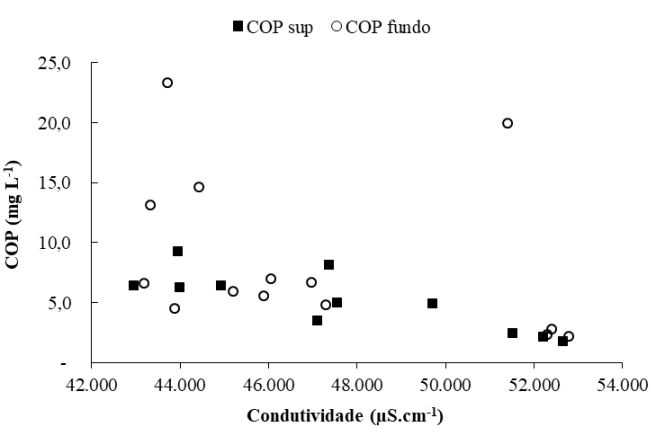

(c) do COD e COP ao longo do gradiente de salinidade que confirma a presença de fontes e sumidouros de $\mathrm{CO}$, podemos afirmar que o CESM apresenta uma contribuição significativa na exportação de carbono para o Atlântico Equatorial adjacente. Comparando a exportação de $\mathrm{C}$ em três bacias de drenagens importantes para a região nordeste do Brasil, Carvalho et al. (2017) mostrou que as concentrações de COD na Plataforma Continental à frente do CESM variaram de 0,48 a $0,75 \mathrm{mg} \mathrm{L}^{-1}$ enquanto nas regiões de plataforma em frente ao rios Parnaíba e Jaguaribe, variaram entre $0,70 \mathrm{mg} \mathrm{L}^{-1}$ e 0,58 . Esse comportamento de relativa diminuição das concentrações de carbono na interface continente-oceano é esperado, já que os valores no oceano são inferiores aos encontrados na zona costeira, e estes, menores que os encontrados nas fontes terrestres, evidenciando o avanço e permanência da massa de água costeira no CESM durante a estação seca. Moyer et al., (2015) observaram

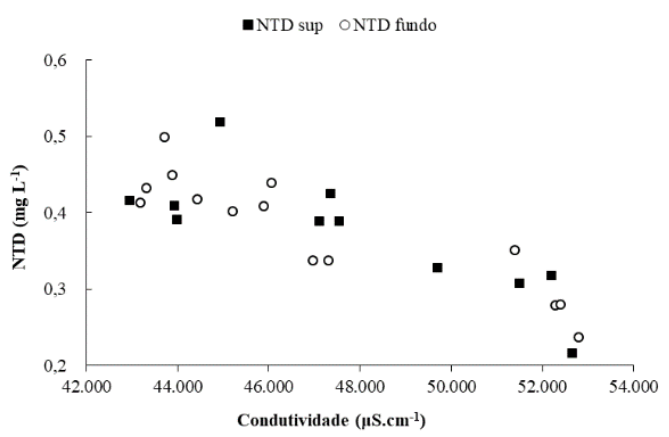

(b)

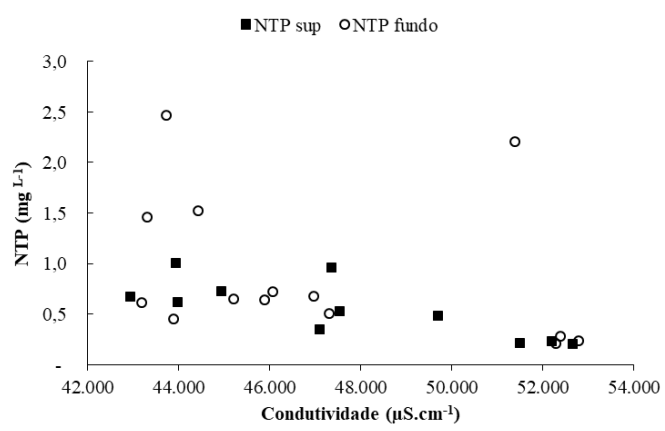

(d)

Figura 3

(c) COP e (d)NTP em relação a condutividade. Os símbolos quadrados fechados indicam as concentrações na superfície e o símbolo circular aberto indicam as concentrações no fundo.

\subsection{A COMPOSIÇÃO ELEMENTAR (\%), RAZÃO ATÔMICA C/N(A) ISOTÓPICA (\%o) DA COP E COD}

A composição elementar, a razão atômica $(\mathrm{C} / \mathrm{N})$ a e a isotópica $\left(\delta^{13} \mathrm{C}\right.$ e $\left.\delta^{15} \mathrm{~N}\right)$ da matéria orgânica particulada e dissolvida são apresen- diferenças significativas espaciais e temporais na abundância e fluxo de COD, em cinco pequenos rios subtropicais e riachos de maré em Tampa Bay Estuary, na região costeira e dentro das suas zonas de mistura devido à dinâmica estuarina local. Suzuki et al (2015) mostraram uma ampla variação do COP e COD na região entre a Plataforma Continental (PC) e Margem Continental (MC) entre as isóbatas de 25 a $3000 \mathrm{~m}$. No entanto, quando comparados os intervalos de concentração para o COD variaram de 0,65 a $1,10 \mathrm{mg} \mathrm{L}^{-1}$ na $\mathrm{PC} \mathrm{e}$ 0,52 a $1,06 \mathrm{mg} \mathrm{L}^{-1}$ na $\mathrm{MC}$, em ambas regiões, o COP esteva abaixo de $8 \%$ do carbono orgânico total.

A relação entre carbono orgânico e o nitrogênio total tanto na fração particulada $\left(\mathrm{r}^{2}=0,888\right)$ quanto na dissolvida $\left(\mathrm{r}^{2}=0,950\right)$ foram significativas $(\mathrm{p}<0,01)$. No entanto, na fração particulada há contribuição da forma inorgânica de nitrogênio enquanto na fração dissolvida o nitrogênio é basicamente orgânico (Figura 4). taram variações entre as áreas de coleta. 


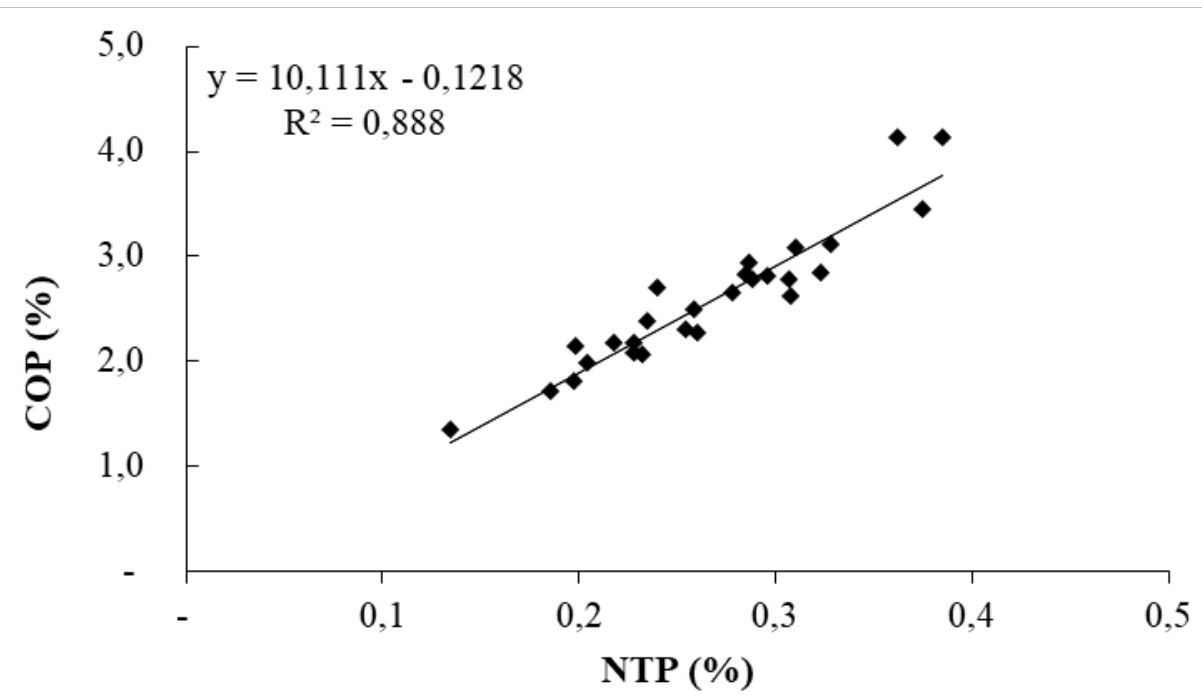

(a)

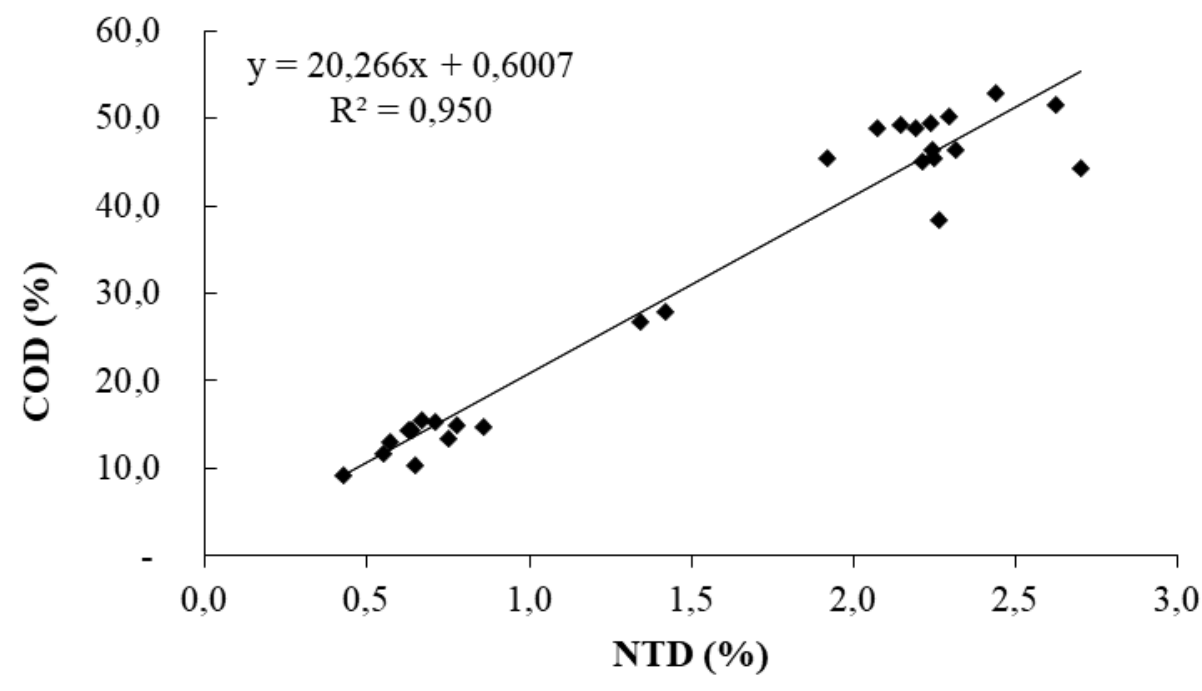

(b)

Figura 4

(a) relação entre carbono orgânico particulado e nitrogênio particulado, em ambas as profundidades de coleta e com todos os pontos amostrais. (b) plotagem carbono orgânico dissolvido e nitrogênio orgânico dissolvido com todos os pontos amostrais e suas respectivas profundidades.

Os valores de $\delta^{13} \mathrm{C}$ em todo o complexo estuarino a COP superficial e de fundo variou entre $-27,7 \%$ a $-25,8 \%$ e $-28,4 \%$ a $-25,8 \%$, respectivamente. As assinaturas isotópicas do COD variaram de $-26,1 \%$ a $-24,4 \%$ na superfície da coluna d'água, enquanto na amostra de fundo não foram observadas variações nas assinaturas, valores próximos a $25,4 \%$. O $\delta^{15} \mathrm{~N}$ na superfície da coluna d'água para o NTP e NTD apresentaram valores de $1,6 \%$ a $5,9 \%$ e de $1,9 \%$ a $4,9 \%$, respectivamente. Na porção inferior da coluna de água as assinaturas $\delta^{15} \mathrm{~N}$ para a forma particulada e dissolvida variaram de $1,0 \%$ a $4,4 \%$ e de 1,7 a $5,8 \%$, respectivamente.
A razão atômica $(\mathrm{C} / \mathrm{N})$ a da matéria orgânica dissolvida na água superficial teve média de 24 e nas amostras de fundo a média foi de 25 . As médias da razão $(\mathrm{C} / \mathrm{N})$ a para a matéria orgânica dissolvida foram de 10 e 11 nas amostras superficiais e de fundo, respectivamente. Indicando que nessa baía ocorre mistura de fontes, e a produção primária contribui para a fase particulada, enquanto a decomposição bacteriana do material terrestre pode estar contribuindo para a fase dissolvida.

Segundo Meyers (1997), um valor de C/N $>20$ é um indicativo de forte contribuição de fontes terrestres oriunda de plantas superiores, uma vez que apresentam em sua composição 
Tabela 2. - Composição elementar, isotópica e razão (C/N)a, (a) na MOP na superfície, (b) MOP na água de fundo, (c) MOD na coluna de água superficial e (d) MOD na porção inferior da coluna d'água no complexo estuarino da Baía de São Marcos.

\begin{tabular}{|c|c|c|c|c|c|c|c|c|c|c|c|}
\hline \multirow{2}{*}{\multicolumn{2}{|c|}{ Pontos }} & \multicolumn{5}{|c|}{ Fase Particulada } & \multicolumn{5}{|c|}{ Fase Dissolvida } \\
\hline & & $\mathrm{C}(\%)$ & $N(\%)$ & $\delta C 13$ & $\delta \mathrm{N} 15$ & $(\mathrm{C} / \mathrm{N}) \mathrm{a}$ & $\mathrm{C}(\%)$ & $\mathrm{N}(\%)$ & $\delta \mathrm{C} 13$ & $\delta \mathrm{N} 15$ & $(\mathrm{C} / \mathrm{N}) \mathrm{a}$ \\
\hline \multirow{14}{*}{ 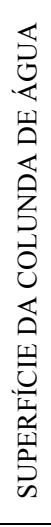 } & 1 & 2,17 & 0,23 & $-25,9$ & 2,8 & 11 & 14,30 & 0,63 & $-25,2$ & 4,8 & 27 \\
\hline & 2 & 2,37 & 0,24 & $-27,1$ & 2,8 & 12 & 11,74 & 0,55 & $-26,0$ & 4,9 & 25 \\
\hline & 3 & 1,71 & 0,19 & $-26,5$ & 3,2 & 11 & 14,76 & 0,86 & $-24,4$ & 4,0 & 20 \\
\hline & 4 & 2,06 & 0,23 & $-26,0$ & 2,9 & 10 & 14,33 & 0,63 & $-25,5$ & 3,8 & 26 \\
\hline & 5 & 2,61 & 0,31 & $-25,9$ & 2,5 & 10 & 15,29 & 0,71 & $-25,4$ & 3,0 & 25 \\
\hline & 6 & 1,34 & 0,13 & $-27,4$ & 2,5 & 12 & 10,29 & 0,65 & $-24,8$ & 2,1 & 19 \\
\hline & 7 & 1,80 & 0,20 & $-26,4$ & 2,0 & 11 & 48,96 & 2,19 & $-25,7$ & 2,4 & 26 \\
\hline & 8 & 2,47 & 2,25 & $-26,0$ & - & - & 49,25 & 2,14 & $-26,1$ & 4,1 & 27 \\
\hline & 9 & 4,12 & 0,39 & $-26,2$ & 5,4 & 12 & 44,21 & 2,70 & $-25,5$ & 2,4 & 19 \\
\hline & 10 & 2,65 & 0,28 & $-27,6$ & 3,3 & 11 & 52,81 & 2,44 & $-25,9$ & 1,9 & 25 \\
\hline & 11 & 4,12 & 0,36 & $-26,2$ & 5,9 & 13 & 46,40 & 2,24 & $-25,2$ & 2,3 & 24 \\
\hline & 12 & 2,94 & 0,29 & $-27,7$ & 2,3 & 12 & 45,50 & 2,25 & $-24,9$ & 2,4 & 24 \\
\hline & 13 & 3,44 & 0,38 & $-26,0$ & 2,3 & 11 & 38,38 & 2,27 & $-24,7$ & 2,9 & 20 \\
\hline & 14 & $, 2,83$ & 0,32 & $-25,8$ & 3,6 & 10 & 27,87 & 1,42 & $-24,6$ & 2,5 & 23 \\
\hline \multirow{14}{*}{ 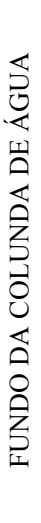 } & 1 & 2,13 & 0,20 & $-26,9$ & 2,7 & 13 & 13,02 & 0,57 & $-25,4$ & 4,7 & 27 \\
\hline & 2 & 2,16 & 0,22 & $-26,6$ & 1,3 & 12 & 15,42 & 0,67 & $-25,4$ & 4,3 & 27 \\
\hline & 3 & 3,11 & 0,33 & $-26,6$ & 3,3 & 11 & 14,37 & 0,64 & $-25,5$ & 3,6 & 26 \\
\hline & 4 & 2,06 & 0,23 & $-25,8$ & 2,0 & 11 & 13,39 & 0,75 & $-25,4$ & 3,9 & 21 \\
\hline & 5 & 1,97 & 0,20 & $-26,7$ & 2,1 & 11 & 14,89 & 0,78 & $-25,1$ & 2,7 & 22 \\
\hline & 6 & 2,27 & 0,26 & $-26,5$ & 1,0 & 10 & 9,17 & 0,42 & $-25,1$ & 3,0 & 25 \\
\hline & 7 & 2,29 & 0,25 & $-25,7$ & 2,2 & 11 & 51,62 & 2,62 & $-25,8$ & 2,1 & 23 \\
\hline & 8 & 2,77 & 0,29 & $-25,9$ & 2,6 & 11 & 49,39 & 2,24 & $-25,9$ & 5,8 & 26 \\
\hline & 9 & 2,82 & 0,29 & $-27,2$ & 4,2 & 12 & 50,18 & 2,29 & $-25,7$ & 2,2 & 26 \\
\hline & 10 & 2,48 & 0,26 & $-27,0$ & 4,1 & 11 & 45,14 & 2,21 & $-25,6$ & 2,2 & 24 \\
\hline & 11 & 2,69 & 0,24 & $-28,4$ & 3,5 & 13 & 45,46 & 1,92 & $-25,1$ & 4,2 & 28 \\
\hline & 12 & 2,78 & 0,31 & $-26,1$ & 2,9 & 11 & 48,93 & 2,07 & $-25,5$ & 1,7 & 28 \\
\hline & 13 & 3,07 & 0,31 & $-25,7$ & 4,4 & 12 & 26,68 & 1,34 & $-25,3$ & 2,4 & 23 \\
\hline & 14 & 2,79 & 0,30 & $-26,9$ & 3,3 & 11 & 46,43 & 2,31 & $-25,2$ & 3,2 & 23 \\
\hline
\end{tabular}

altos conteúdos de celulose, e consequentemente, altos teores de carbono. De Souza et al., (2017) e Liu et al (2015) apontam que variações nos valores de razão $\mathrm{C} / \mathrm{N}$ encontrados nos ambientes costeiros são indicativos das diferentes fontes de MO assim como o aporte de compostos nitrogenados. Neste sentido, foram coletadas amostras de sedimento, folhas de mangue e material particulado em suspensão na região da baía de São Marcos e as razões atômicas $(\mathrm{C} / \mathrm{N})$ a foram de $9,9 \pm 2,4 ; \quad 117,4 \pm 29,2 ; \quad$ e $11,6 \pm 0,9$, respectivamente (Serejo, 2018). Considerando o exposto, a razão atômica $(\mathrm{C} / \mathrm{N})$ a não traz propriamente um indicativo da vegetação vascular terrestre, mas tipifica uma contribuição marinha no material particulado em suspensão e nos sedimentos do manguezal. No entanto, ao determinarmos a composição isotópica do carbono orgânico nos mesmos compartimentos (sedimento $=-24,2 \pm 0,9 \%$; folhas de mangue $=-28,2 \pm 0,6 \%$; material particulado em suspensão $=-24,3 \pm 0,6 \%$ ) observa-se uma mistura de fontes nos sedimentos e material particulado em suspensão, uma vez que, o fitoplâncton marinho possui valores do $\delta^{13} \mathrm{C}$ em torno de 20,0\%o (Rezende et al, 2010).

A quantificação relativa da contribuição marinha e do manguezal na baía nas formas de COP e COD na água foi estimada através de um modelo de duas fontes considerando os manguezais e o fitoplânton marinho seguindo modelo descrito por Rezende et al. (1990). Desta forma, consideramospara o manguezal o ${ }^{13} \mathrm{C}$ com o valor médio de $-28,2 \%$ (Serejo 2018) e para a fonte marinha o ${ }^{13} \mathrm{C}$ de $-20,0 \%$ (Rezende et al, 2010). A contribuição do manguezal no COP foi entre 71 a $94 \%$ e71 a $100 \%$ nas águas de superfície e fundo respectivamente, enquanto no COD a contribuição do manguezal foi de 54 a $73 \%$ na superfície e 62 a $72 \%$ no fundo.

Segundo Kristensen et al., (2008) 58\% dos seus dados compilados mostraram que a $\delta^{13} \mathrm{C}$ são inferiores a $-25 \%$ e, portanto, sugerem uma entrada importante de detritos de manguezal $\left(\delta^{13} \mathrm{C} \sim-28 \%\right.$ a $-30 \%$ ). Middelburg \& Herman (2007) estudando os processos da matéria orgânica em estuários de maré, observaram que estuários de alta turbidez encontraram valores do $\delta^{13} \mathrm{C}$ variando entre$30 \%$ e $-22 \%$. Em um estuário localizado na 
região Norte do Brasil, Giarrizzo et al. (2011) observaram que a fonte de carbono predominante na fase particulada vinha de detritos de manguezal, principalmente folhas onde $o \delta^{13} \mathrm{C}$ apresentou uma faixa estreita de variação entre $-25,5$ e $-25,2 \%$.

Os resultados obtidos da $\delta^{13} \mathrm{C}$ e as características de maré local são condizentes com alguns estudos (Rezende et al. 1993, Dittmar 2001, Hermes \& Sikes 2016, Sigma et al. 2017) nos quais manguezais dominados pelo bombeamento de maré são caracterizados por fluxos bidirecionais que favorecem a retenção do material autóctone e fontes marinhas. As marés de sizígia favorecem a ressuspensão e transporte de massa da matéria orgânica dos manguezais, os quais aumentam a exportação para a plataforma continental adjacente (Rezende et al. 1993).

O presente estudo, apresentou similaridade ao estudo Bardhan et al. (2015) que encontrou teores de $\delta^{15} \mathrm{~N}$ variando de $1,0 \%$ a $6,5 \%$, reforçando as misturas de fontes, mas os menores valores da composição isotópica do nitrogênio se relacionam ao amônio que sai do sedimento através do processo de amonificação gerando uma matéria orgânica empobrecida de ${ }^{15} \mathrm{~N}$. Outra fonte de variabilidade dos valores de $\delta^{15} \mathrm{~N}$ é a contribuição continental, limitação de nitrogênio no sistema marinho refletida na composição isotópica do fitoplâncton da região assim como alteração durante a decomposição da matéria orgânica que gera a proliferação de espécies que fixam nitrogênio atmosférico em situação de escassez de nitrogênio modificando o sinal do $\delta^{15} \mathrm{~N}$ e reforçando assim a necessidade de estudarmos com mais atenção a comunidade fitoplanctônica (Maya et al. 2011). Em síntese, os resultados reportados nesse estudo em relação à composição isotópica e razão atômica $(\mathrm{C} / \mathrm{N})$ a do carbono orgânico particulado e dissolvido foram semelhantes aos estudos apresentados por outros autores apontando pouca variabilidade na composição isotópica do carbono orgânico das espécies vegetais submetidas a diferentes tipologias dos manguezais, aportes fluviais e gradientes latitudinais (Tabela 3 ).

Tabela 3 - Composição isotópica do COP e COD em alguns ambientes estuarinos

\begin{tabular}{cccc}
\hline Local & $\boldsymbol{\delta}^{\mathbf{1 3}} \mathbf{C ~ ( \% )}$ & $(\mathbf{C} / \mathbf{N}) \mathbf{a}$ & Referências \\
\hline Baía de Sepetiba & $-26,7 \pm 0,5$ & - & Rezende et al. 1990 \\
WinyahBay & $-28,0 \mathrm{a}-25,0$ & 8 a 32 & Goñiet al. 2003 \\
Baía de Babitonga & $-26,2$ a $-21,7$ & 6 a 13 & Barros et al. 2010 \\
Yellow River Estuary & $-23,9$ a $-20,8$ & 6 a 12 & Liu et al. 2015 \\
Rio Mocajuba & $-27,7 \pm 0,7$ & $6 \pm<1$ & Vilhena et al. 2017 \\
Rio Pará & $-26,4 \pm 0,6$ & $8 \pm 1$ & Vilhena et al. 2017 \\
Baía de São Marcos & $-28,4$ a $-24,4$ & 10 a 28 & Presente estudo \\
\hline
\end{tabular}

\section{CONCLUSÃO}

O carbono orgânico dissolvido representou em média maior quantidade no fundo do que na superfície do carbono orgânico total transportado na região do Complexo Estuarino de São Marcos (CESM), tendo uma assinatura tipicamente de matéria orgânica continental. A razão atômica $(\mathrm{C} / \mathrm{N}) \mathrm{a}$ indica a liberação do nitrogênio e humificação da matéria orgânica dissolvida proveniente da vegetação vascular. A média da composição isotópica $\left(\delta^{13} \mathrm{C}\right)$ do carbono orgânico particulado e dissolvido (COP e COD) na superfície e fundo foi de $26,6 \%$ e $-25,4 \%$, respectivamente. Portanto, atribuímos como principal fonte de matéria orgânica o ecossistema dos manguezais que circundam o CESM, mas reconhecimento à necessidade de ampliar os estudos referentes aos aportes continentais, principalmente através do rio Mearim, assim como realizar um inventário isotópico da bacia de drenagem deste sistema fluvial. O desenho experimental nos permitiu demonstrar que há uma remoção das formas particuladas e dissolvidas de carbono e nitrogênio da massa de água por processos de floculação ao longo do gradiente de força iônica, mas o CESM representa um importante aporte de matéria orgânica para o Atlântico Equatorial aonde ainda existem poucas informações quando comparado com outras regiões da Costa Brasileira. 


\section{AGRADECIMENTO}

Os autores agradecem a Fundação de Amparo à Pesquisa do Estado do Maranhão (FAPEMA, Edital Infraestrutura 03894/15; Edital Universal - 00112/16) e financiamento institucional (PRONEM - Processo: 00079/16). Ao Conselho Nacional de Desenvolvimento Científico e Tecnológico (CNPq) pelo financiamento individual (Edital Universal Processo: 471924 / 2013-0) concedido a Francisco José da Silva Dias e ao Instituto Nacional de Ciência e Tecnologia de Transferências de Materiais Continente Oceano (INCT-TMCOcean - 573601 / 20089).Ao Laboratório de Biogeociclos dos Constituintes Químicos da Água

\section{REFERÊNCIAS}

Abrantes et al. 2014, - Abrantes KG, Johnston R, Connolly RM, Sheaves, M. 2014. Importance of Mangrove Carbon for Aquatic Food Webs in Wet-Dry Tropical Estuaries. Estuaries and Coasts. 38: 383-399.

Araujo TD. Boletim Climático do Maranhão. Em: Sagrima, Boletim Informativo, Governo do Estado do Maranhão, 2017

Bardhan P, Karapurkar SG, Shenoy DM, Kurian S, Sarkar A, Maya MV, Naqvi SWA. 2015. Carbon and nitrogen isotopic composition of suspended particulate organic matter in Zuari Estuary, west coast of India. Journal of Marine Systems, 141, 90-97.

Barros GV, Martinelli LA, Oliveira Novais TM, Ometto JPHB, Zuppi GM. 2010. Stable isotopes of bulk organic matter to trace carbon and nitrogen dynamics in an estuarine ecosystem in Babitonga Bay (Santa Catarina, Brazil). Science of the Total Environment. 408: 2226-2232.

Bauer JE, Cai WJ, Raymond PA, Bianchi TS, Hopkinson CS, Regnier PAG. 2013. The changing carbon cycle of the coastal ocean. Nature. 504: 61-70.

Bianchi, TS. 2006. Biogeochemistry of estuaries. Oxford University Press.721 p.

Bouillon S, Chandra MP, Sreenivas N, Dehairs F. 2000. Sources of suspended organic matter and selective feeding by zooplankton in an estuarine mangrove ecosystem as traced by stable isotopes. Marine Ecology Progress Series, 208: 79-92.

Bouillon S, Connolly RM, Lee SY. 2008. Organic matter exchange and cycling in mangrove ecosystems: Recent insights from stable isotope studies. Journal of Sea Research. 59: 44-58

Bouillon S, Connolly RM, Gillikin DP. 2011. 7.07 Use of Stable Isotopes to Understand Food Webs and Ecosystem Functioning in Estuaries. Treatise on Estuarine and Coastal Science, 7.
(Labciclos/UFMA) por disponibilizar o laboratório para a realizarmos as filtragens e separações das alíquotas amostras. Ao Laboratório de Ciências Ambientais (LCA/UENF) e Carlos Eduardo de Rezende é membro do Future Earth Coastshttps://www.futureearthcoasts.org/south -america-rep/) e recebe apoio financeiro do CNPq (305217/2017-8) e FAPERJ (E26/202.916/2017). O presente trabalho foi realizado com apoio da Coordenação de Aperfeiçoamento de Pessoal de Nível Superior - Brasil (CAPES) - Código de Financiamento 001.

Bristow LA, Jickells TD, Weston K, Marca-Bell A, Parker R, Andrews JE. 2013. Tracing estuarine organic matter sources into the southern North Sea using $\mathrm{C}$ and $\mathrm{N}$ isotopic signatures. Biogeochemistry. 113: 9-22.

Canuel EA. 2001. Relations between river flow, primary production and fatty acid composition of particulate organic matter in San Francisco and Chesapeake Bays: a multivariate approach. Organic Geochemistry. 32: 563-583,

Canuel EA \& Hardison AK. 2016. Sources, Ages, and Alteration of Organic Matter in Estuaries. Annual Review of Marine Science. 8: 409-434.

Carvalho ACO, Marins RV, Dias FJS, Rezende CE, Lefèvre N, Cavalcante MS, Eschrique SA. 2017. Air-sea CO2 fluxes for the Brazilian northeast continental shelf in a climatic transition region. Journal of Marine Systems. 173: 70-80.

Dias FJS, Castro BM, Lacerda LD. 2013. Continental shelf water masses off the Jaguaribe river $\left(4^{\circ} \mathrm{S}\right)$, Northeastern Brazil. Continental Shelf Research, 66: 123 - 135.

Dittmar T \& Lara RJ. 2001. Driving Forces Behind Nutrient and Organic Matter Dynamics in a Mangrove Tidal Creek in North Brazil. Estuarine, Coastal and Shelf Science. 52: 249259.

Dittmar T, Hertkorn N, Kattner G, Lara RJ. 2006. Mangroves, a major source of dissolved organic carbon to the oceans. Global Biogeochemical Cycles. 20: 1-7.

Dittmar T, Koch B, Hertkorn N, Kattner G. 2008. A simple and efficient method for the solid-phase extraction of dissolved organic matter (SPEDOM) from seawater. Limnology and Oceanography Methods. 6: 230-235.

EL-Robrini M et al. 2006. Maranhão. Erosão e progradação do litoral brasileiro: Maranhão. Brasília, MMA. Access, v. 14, n. 10, 2014p 
Eckert JM, Sholkovitz ER. 1976. The floculation of iron, aluminium and humates from river water by electrolytes. Geochimica et Cosmochimica Acta. 40: 847-848.

FAO (Food and Agriculture Organization of the United Nations). 2007. The world's mangroves 1980-2005. FAO Forestry Paper. 153

Fry B. 1981. Natural stable carbon isotope tag traces Texas shrimp migrations. Fish Bull, 79: 337-45.

Giarrizzo T, Schwamborn R, Saint-Paul U. 2011. Utilization of carbon sources in a northern Brazilian mangrove ecosystem. Estuarine, Coastal and Shelf Science. 95: 447-457.

Giri C. et al. 2011. Status and Distribution of Mangrove Forests of the World Using Earth Observation Satellite Data. Global Ecology and Biogeography. 20:154-59.

Goñi MA, Teixeira MJ, Perkeya DW. 2003. Sources and distribution of organic matter in a river-dominated estuary (Winyah Bay, SC, USA). Estuarine, Coastal and Shelf Science. 57:1023-1048

Hedges JI, Keil RG, Benner R. 1997. What happens to terrestrial organic matter in the ocean. Organic Geochemistry 27:195-212

Hermes AL. \& Sikes EL. 2016. Particulate Organic Matter Higher Concentrations, Terrestrial Sources and Losses in Bottom Waters of the Turbidity Maximum, Delaware Estuary, U.S.A. Estuarine, Coastal and Shelf Science 180:17989

Hertkonet al. 2002 - Hertkon N, Claus H, SchmittKopplin PH, Perdue E.M. 2002 Utilization and transformation of aquatic humic substances by autochthonous microorganisms. Environmental Science and Technology. 36: 4334-4345.

Hobbie J.E. (ed.) 2000. Estuarine science: the key to progress in coastal ecological research. In Estuarine Science: A Synthetic Approach to Research and Practice. Island Press, Washington, DC, 526pp.

Kristensen E, Bouillon S, Dittmar T, Marchand C. 2008. Organic carbon dynamics in mangrove ecosystems: A review. Aquatic Botany. 89: 201-219.

Lacerda LD, Rezende CE de, Martinelli LA, Ovalle ARC, Mozeto AA, Nogueira F, Victoria RL, Aragon GT, Cunha CT da. 1986. Composição isotópica de carbono em componentes de um ecossistema de manguezal, Baía de Sepetiba, RJ. Ciência e Cultura. 38: 1714-1717.

Levèvre N, Dias FJS, Torres Jr AR, Noriega C, Araújo M, Castro ACL, Rocha C, Jiang S, Ibánhez JSP. A source of $\mathrm{CO}_{2}$ to the atmosphere throughout the year in the Maranhense continental shelf $\left(2^{\circ} 30^{\prime} \mathrm{S}\right.$, Brazil). Continental Shelf Research, 141: $38-50$.

Li Y, Zhang H, Tu C, Fu C, Xue Y, Luo Y. 2016. Sources and fate of organic carbon and nitrogen from land to ocean: Identified by coupling stable isotopes with $\mathrm{C} / \mathrm{N}$ ratio. Estuarine Coastal and Shelf Science. 181: 114-122.
Liu D, Li X, Emeis KC, Wang Y, Richard P. 2015. Distribution and sources of organic matter in surface sediments of Bohai Sea near the Yellow River Estuary, China. Estuarine, Coastal and Shelf Science, 165: 128-136.

Luz DS, Pinheiro RMP, Bezerra, VLAR. 2005. Panorama da Desertificação do Estado do Maranhão. 2005

Mari X, Torréton JP, Trinh, CBT, Bouvier T, Chu, VT, Lefebvre JP, Ouillon S. Aggregation dynamics along a salinity gradient in the Bach Dang estuary, North Vietnam. Estuarine, Coastal and Shelf Science. 96: 151-158.

Martinelli LA, Ometto JPHB, Ferraz ES, Victoria RL, Camargo PB, Moreira MZ. 2009 Desvendando questões ambientais com isótopos estáveis. São Paulo. Oficina de Textos. p.144.

Maya MV, Soares MA, Agnihotri R, Pratihary AK, Karapurkar S, Naik H, Naqvi SWA. 2011. Variations in some environmental characteristics including $\mathrm{C}$ and $\mathrm{N}$ stable isotopic composition of suspended organic matter in the Mandovi Estuary. Environ. Monit. Assess. 175: 501-517.

Mctiernanet al. 2001, - Mctiernan, K.B, Jarvis, S.C, Scholefield, D, Hayes M.H.B. 2001 Dissolved organic carbon losses from grazed grasslands under different management regimes. Water Research. 35: 2565-2569

Meyers P. 1994. Preservation of elemental and isotopic source identification of sedimentary organic matter. Chemical Geology. 114: 289302.

Meyers PA. 1997. Organic geochemical proxies of paleoceanographic, paleolimnologic, and paleoclimatic processes. Organic geochemistry. 27: 213-250.

Middelburg JJ \& Herman PMJ. 2007. Organic matter processing in tidal estuaries. Marine Chemistry. 106: 127-147.

Mikkelsen OA, Hill PS, Milligan TG. 2007. Seasonal and spatial variation of floc size, settling velocity and density on the inner Adriatic Shelf (Italy). Continental Shelf Research. 27: 417-430.

Miranda LB, Castro BM, Kjerfve B. 2002. Princípios de oceanografia física de estuários. EDUSP. São Paulo, p.411.

Moyer RP, Bauer JE, Grottoli AG. 2013. Carbon isotope biogeochemistry of tropical small mountainous river, estuarine, and coastal systems of Puerto Rico. Biogeochemistry, 112(1-3), 589-612.

Moyer RP, Powell CE, Gordon DJ, Long JS, Bliss CM. 2015. Abundance, distribution, and fluxes of dissolved organic carbon (DOC) in four small sub-tropical rivers of the Tampa Bay Estuary (Florida, USA). Applied Geochemistry. 63: 550-562.

Pancost RD, Boot CS. 2004. The palaeoclimatic utility of terrestrial biomarkers in marine sediments. Marine Chemistry. 92: 239-261. 
Rezende CE, Lacerda LD, Ovalle ARC, Silva CARE, Martinelli LA. 1990. Nature of POC transport in a mangrove ecosystem: A carbon stable isotopic study. Estuarine, Coastal and Shelf Science. 30: 641-646

Rezende, CE, Lacerda, LD, Ovalle, ARC, Silva, LFF. 2007, Dial organic carbono fluctuations in a mangrove tidal creek in Sepetiba bay, Southeast Brazil, Brazilian Journal of Biology, 67(4): $673-680$.

Serejo JHF. 2018. Desvendando as fontes de matéria orgânicapara os sedimentos de uma área de manguezal sob a influência de macromaré: Complexo Estuarino de São Marcos. Dissertação de Mestrado, Programa de PósGraduação em Oceanografia, Universidade Federal do Maranhão. 64 p.

Signa G, Mazzola A, Kairo J, Vizzini S. 2017. Small-scale variability in geomorphological settings influences mangrove-derived organic matter export in a tropical bay. Biogeosciences. 14: 617-629

Souza JRB de, Rosário ZM do, Costa AB, Azevedo AEG de, Spano S. 2017. Geochemical markers of sedimentary organic matter in Todosos Santos Bay, Bahia-Brazil. Indicators of sources and preservation. Marine Pollution Bulletin. 119: 239-246.

Souza, TA, Godoy, JM, Godoy, MLDP, Moreira, I, Carvalho, Z, Salomão, MSMB, Rezende, CE. 2010. Use of multitracers for the study of water mixin in the Paraíba do Sul river estuary.Journal of Environmental Radioactivity, 101: 564 - 570.

Suzuki MS, Rezende CE, Paranhos R, Falcão AP. 2015. Spatial distribution (vertical and horizontal) and partitioning of dissolved and particulate nutrientes (C, N and $\mathrm{P}$ ) in Campos Basin, Southern Brazil. Estuarine, Costal and Shelf Science, 166: 4-12.

Teixeira SG \& Souza Filho PWM. 2009. Mapeamento de Ambientes Costeiros Tropicais (Golfão Maranhense, Brasil) Utilizando Imagens de Sensores Remotos Orbitais. Revista Brasileira de Geofísica (impresso). 27: 69-82.

Tremblay LB, Dittmar T, Marshall AG, Cooper WJ, Cooper WT. 2007. Molecular characterization of dissolved organic matter in a North Brazilian mangrove porewater and mangrove-fringed estuaries by ultrahigh resolution Fourier Transform-Ion Cyclotron Resonance mass spectrometry and excitation/emission spectroscopy. Marine Chemistry. 105: 15-29. 